\title{
Efecto de la aplicación de IBA sobre el enraizamiento de estacas en seis especies arbustivas nativas de la región mediterránea de Chile
}

\author{
Effect of IBA application on rooting of semi hard wood cuttings of six \\ native shrubs from the mediterranean area of Chile
}

Ursula Doll ${ }^{1 *}$, Catherine Norambuena M., Osvaldo Sánchez V.

\section{RESUMEN}

Muchas especies arbustivas de los ambientes áridos y semiáridos chilenos actúan como plantas nodrizas sobre el reclutamiento de propágulos, característica especialmente interesante para utilizarlas como colonizadoras de taludes que faciliten el posterior reclutamiento de especies herbáceas para la formación de una cubierta vegetal estabilizadora. En este contexto se eligieron seis especies arbustivas nativas de la zona mediterránea de Chile, con el propósito de poder utilizarlas para iniciar un proceso de revegetación en taludes, mediante plantas obtenidas por enraizamiento de estacas semileñosas. Se realizaron dos ensayos en invernadero para evaluar el efecto del enraizante en polvo (1500 ppm IBA) sobre el enraizamiento y la supervivencia de las estacas. Las estacas de Escallonia illinita tratadas con hormona alcanzaron un enraizamiento del $73 \%$ y las de Fabiana imbricata del 79\%, al cabo de nueve y doce semanas, respectivamente. Ambas especies se perfilan como promisorias para enraizar directamente en el talud durante la época de lluvias.

Palabras clave: propagación vegetativa, Escallonia illinita, Muehlenbeckia hastulata, Proustia cuneifolia, Fabiana imbricata.

\begin{abstract}
Many shrubs of the arid and semiarid Chilean environments act as nurse plants on propagule recruitment. This characteristic makes them especially amenable to be used as slopes' colonists that facilitate the recruitment of herbs to form a stabilizing vegetal cover. In this context, 6 native shrubs of the mediterranean area of Chile were chosen to be used to initiate a revegetation process in slopes, through rooting of semi hard wood cuttings. 2 glasshouse trials were carried out, to evaluate the influence of commercial rooting hormone (IBA) on rooting and survival of cuttings. Escallonia illinita cuttings treated with hormone reached $73 \%$ rooting and Fabiana imbricata cuttings 79\%, after 9 and 12 weeks, respectively. Both species seem to be promising to root directly on slopes during the rainfall period.
\end{abstract}

Key words: vegetative propagation, Escallonia illinita, Muehlenbeckia hastulata, Proustia cuneifolia, Fabiana imbricata.

\section{Introducción}

Varios son los factores que interactúan en la posibilidad de que una superficie de suelo sea erosionada o no. Así la cantidad, intensidad y distribución estacional de la precipitación, características inherentes al suelo relacionadas con la capacidad de infiltración y estabilidad estructural, la topografía con relación al largo e inclinación de la pendiente y por último la cubierta y el manejo a que está sometido el suelo son los principales factores que influyen en el proceso erosivo (Brady y Weil, 1996; Honorato, 2000).

El establecimiento de una cubierta vegetal perenne sobre la superficie de los taludes permite estabilizarlos y minimizar los problemas que trae consigo la erosión de los mismos. Al aminorar el impacto de las gotas de lluvia que desprenden las partículas de la superficie de suelo descubierto, disminuye el riesgo de inicio del proceso erosivo. A su vez, aumenta la infiltración hídrica al oponer resistencia al escurrimiento superficial y mejorar la estructura del suelo por la incorporación de materia orgánica en el mismo. La proliferación de las raíces a su vez ayuda a anclar y sostener los taludes, impidiendo la producción de movimientos o deslizamientos en masa (Gyssels, 2005).

En este sentido existe amplia experiencia sobre el uso de la vegetación nativa como estabilizadora de terrenos con pendiente y taludes (Belmonte et al.,

\footnotetext{
$1 \quad$ Facultad de Ciencias Forestales, Universidad de Talca, Casilla 721-747, Talca, Chile.

* Autor para correspondencia: udoll@utalca.cl
} 
1999; Soto, 2003; Etxeberria e Ibáñez, 2005; Guevara et al., 2005; Rondón y Vidal, 2005; Matezanz et al., 2006; Bochet et al., 2011).

La flora chilena de la zona central y norte del país integra especies que se destacan por su capacidad de adaptarse a condiciones edáficas extremas y por haber desarrollado estrategias para sobrevivir en condiciones de escasez de agua. Estas características son especialmente deseadas en especies que puedan ser utilizadas para formar cubiertas vegetales restauradoras (Rondon y Vidal, 2005). Por otro lado muchas de las especies arbustivas de los ambientes áridos y semiáridos chilenos presentan el fenómeno del levantamiento hidráulico y actúan como plantas nodrizas sobre el reclutamiento de propágulos (Muñoz et al., 2008; Prieto et al., 2011). Estas características las destacan como colonizadoras de taludes que facilitan el posterior reclutamiento de especies herbáceas y la formación de una cubierta vegetal estabilizadora. Villar-Salvador et al. (2011) sostienen que, especialmente en ambientes mediterráneos, la introducción de especies leñosas acelera la sucesión secundaria. Su sistema radical más profundo, extenso y resistente que el de las especies herbáceas garantiza en el largo plazo la estabilización del suelo, protegiéndolo de la erosión.

En este contexto se eligieron seis especies arbustivas nativas de la zona mediterránea de Chile, con el propósito de poder utilizarlas para iniciar un proceso de revegetación en taludes.

Escallonia illinita C. Presl, de la familia de las Escalloniaceae, es un arbusto perenne, resinoso, fragante, muy frondoso (Riedemann y Aldunate, 2001). Habita suelos pedregosos y arenosos, aunque requiere humedad en el suelo, resiste períodos de sequía. Muehlenbeckia hastulata (Sm.) I.M. Johnst es un arbusto perenne de la familia Poligonaceae. Se comporta como cubresuelo de rápido crecimiento que requiere algo de humedad en el suelo, pero tolera sequías prolongadas (Riedemann y Aldunate, 2001). Baccharis linearis (Ruiz \& Pav.) Pers. y Proustia cuneifolia D. Don. pertenecen a la familia Asteraceae. Habitan suelos secos y pedregosos. Son arbustos de gran tamaño, capaces de crecer en suelos degradados y pendientes pronunciadas. Florecen en verano, siendo el primero dioico y perennifolio, mientras que el segundo, cuyas ramas terminan en espinas, es caducifolio en invierno. Muñoz et al. (2008) evidenciaron el levantamiento hidráulico debajo de plantas de esta especie. Fabiana imbricata Ruiz \& Pav., de la familia Solanaceae, es un arbusto perenne de hojas imbricadas, que florece abundantemente en primavera con pequeñas flores tubulares en el extremo de ramillas laterales. Crece en suelos con buen drenaje y con alta luminosidad. Colliguaja odorifera Mol. es un arbusto monoico de follaje perenne, coriáceo, de la familia de las Euphorbiaceae. Crece en suelos secos e intervenidos (Riedemann y Aldunate, 2001).

Las seis especies seleccionadas se caracterizan por su rusticidad y capacidad de soportar períodos de sequía. El objetivo de este trabajo es determinar la capacidad de enraizamiento de estacas semileñosas de las mismas mediante la aplicación de IBA, con la finalidad de evaluar la factibilidad de utilizarlas para enraizar directamente en taludes.

\section{Material y Métodos}

Para un primer ensayo se recolectaron a comienzos de agosto de 2005 estacas semileñosas de Escallonia illinita, Muehlenbeckia hastulata y Proustia cuneifolia de los alrededores de Talca. Para el segundo ensayo se recolectaron a mediados de septiembre del mismo año estacas de Fabiana imbricata en el sector lago Colbún y estacas de Baccharis linearis y Colliguaja odorifera en los alrededores de Talca. Con el fin de evitar la deshidratación de las estacas, las bases de las mismas fueron defoliadas, envueltas en papel absorbente humedecido y colocadas dentro de bolsas de polietileno para su trasladado en un recipiente aislante. Previo a su instalación se realizó un nuevo corte en la base de las estacas, dejándolas de una longitud de $10 \mathrm{~cm}$ aproximadamente y se eliminaron todas las hojas y ramillas del 2/3 inferior de la estaca. Se lavaron con abundante agua y se desinfectaron con una solución de captan $\left(1 \mathrm{~g} \times \mathrm{L}^{-1}\right.$, $13,04 \mathrm{~g}$ i.a. en $100 \mathrm{~g}$ ). Inmediatamente después se aplicó enraizante comercial en polvo ( $0,15 \mathrm{~g}$ IBA, $6 \mathrm{~g}$ captan en $100 \mathrm{~g}$ ) a la base de la mitad de las estacas de cada especie.

Las estacas fueron instaladas en almacigueras de poliestireno expandido de 104 cavidades, a razón de una estaca por cavidad en una mezcla 1:1 de turba y perlita como sustrato. Las almacigueras se colocaron sobre un cajón de madera lleno de arena gruesa ubicado a $1 \mathrm{~m}$ de altura sobre el suelo dentro de un invernadero de estructura de madera y cubierta de polietileno con aireación lateral y cenital. El riego de tipo niebla intermitente fue provisto por microaspersores regidos por un sistema que se basa 
en el peso del agua sobre una superficie evaporante, asegurándose una humedad ambiente superior al 50\% de humedad relativa. Quincenalmente se realizaron fumigaciones preventivas con fungicida captan $\left(1 \mathrm{~g} \times \mathrm{L}^{-1}, 13,04 \mathrm{~g}\right.$ i.a. en $\left.100 \mathrm{~g}\right)$.

A las nueve semanas de instalado el primer ensayo y a las doce semanas de instalado el segundo ensayo se cuantificó el número de estacas vivas y el número de estacas enraizadas por tratamiento. Estos valores se expresaron en porcentaje de supervivencia y porcentaje de enraizamiento en base al número inicial de estacas para cada repetición. Para el primer ensayo además se cuantificó el número de raíces visibles a cada lado del pan del sustrato, resultando cuatro valores que se promediaron para cada estaca con el fin de estimar un índice de calidad del sistema radical formado.

El primer ensayo fue dispuesto según un modelo en bloques completamente aleatorizados y contó con diez repeticiones para cada combinación de especie y tratamiento, con diez estacas por repetición. El segundo ensayo, también dispuesto en bloques completamente aleatorizados, contó con cuatro repeticiones para cada combinación de especie y tratamiento, con 25 estacas por repetición.

Los resultados de cada ensayo se sometieron a un análisis de varianza, encontrándose una interacción significativa entre especie, tratamiento y bloques $(P \leq 0,05)$, por lo que se realizaron análisis de varianza independientes para cada especie. Para su evaluación los datos expresados en porcentaje fueron modificados mediante la transformación angular y los datos correspondientes al parámetro de calidad de raíces fueron reemplazados por la raíz cuadrada del valor.

\section{Resultados y Discusión}

En el primer ensayo destacó la supervivencia y el porcentaje de enraizamiento alcanzado por Escallonia illinita, $94 \%$ y $72 \%$ respectivamente. Se trata de una especie de fácil enraizamiento, que incluso según los resultados obtenidos no requiere de aplicación de hormona para promover la formación de raíces (Tabla 1). La alta tasa de supervivencia observada sugiere que posiblemente hubiera aumentado el porcentaje de enraizamiento de prolongarse el tiempo de duración del ensayo. Destacó también la calidad del sistema radical formado por esta especie, ya que los valores alcanzados indican que en promedio fue posible contabilizar más de ocho raíces visibles en el exterior del pan de sustrato. Se ha reportado que Escallonia illinita es capaz de formar raíces en las ramas que apoyan en el suelo, por lo que se la indica como apropiada para proteger pendientes y taludes plantándola en sus cabeceras para que las ramas colgantes arraiguen y protejan el suelo de la erosión (Riedemann y Aldunate, 2001).

El factor determinante en el bajo porcentaje de enraizamiento alcanzado por Muehlenbeckia hastulata (Tabla 1) fue el alto porcentaje de mortandad que presentaron las estacas de esta especie y que llegó a un 64\%. Se observó que sobrevivieron y enraizaron las estacas de mayor grosor. Un factor importante en el éxito de enraizamiento de estacas es la disponibilidad de nutrientes y carbohidratos provenientes de las reservas acumuladas en las estacas o de las hojas que mantienen (Hartmann, et al., 1990, Gutiérrez, 1995). Muehlenbeckia hastulata posee ramas volubles, con entrenudos largos y hojas

Tabla 1. Efecto de la aplicación de hormona comercial (IBA) sobre el enraizamiento [\%] y la supervivencia [\%] de estacas y calidad de raíces formadas en estacas de Escallonia illinita, Muehlenbeckia hastulata y Proustia cuneifolia.

\begin{tabular}{lcccc}
\hline \multicolumn{1}{c}{ Especie } & Tratamiento & $\begin{array}{c}\text { Enraizamiento } \\
{[\%]}\end{array}$ & $\begin{array}{c}\text { Supervivencia } \\
{[\%]}\end{array}$ & $\begin{array}{c}\text { Calidad de raíces } \\
\text { formadas }^{1}\end{array}$ \\
\hline Escallonia illinita & con hormona & $73 \pm 17,67$ & $93 \pm 6,75$ & $2,74 \pm 1,38$ \\
& sin hormona & $71 \pm 19,69$ & $95 \pm 7,07$ & $2,42 \pm 1,29$ \\
Muehlenbeckia hastulata & con hormona & $29 \pm 19,12$ & $34 \pm 21,71$ & $1,17 \pm 0,72$ \\
& sin hormona & $26 \pm 24,13$ & $38 \pm 26,16$ & $0,96 \pm 0,85$ \\
Proustia cuneifolia & con hormona & $45 \pm 21,73 \mathrm{a}$ & $88 \pm 10,33$ & $2,20 \pm 1,44 \mathrm{a}$ \\
& sin hormona & $26 \pm 22,21 \mathrm{~b}$ & $87 \pm 11,60$ & $1,16 \pm 1,02 \mathrm{~b}$ \\
\hline
\end{tabular}

Los valores seguidos de distintas letras en la columna presentan diferencias estadísticamente significativas, según prueba de Rangos Múltiples de Duncan, con $P \leq 0,05$.

${ }^{1}$ Promedio de número raíces visibles en los cuatro lados del pan de sustrato de cada estaca. 
relativamente pequeñas. En el ensayo se utilizaron estacas pequeñas, que no contenían más de una a dos hojas. Una menor disponibilidad de reservas acumuladas en las estacas más delgadas explicaría su mayor mortandad.

A pesar de notarse una mejora en la capacidad de enraizamiento y en la calidad de las raíces formadas en respuesta a la aplicación de hormona (Tabla 1) en ambas especies, esta tendencia no fue significativa debido a la alta variabilidad encontrada entre repeticiones.

Proustia cuneifolia mostró un alto porcentaje de supervivencia que alcanzó cerca del $88 \%$, pero que, sin embargo, no repercutió en el porcentaje de enraizamiento. La aplicación de hormona favoreció significativamente el arraigamiento de las estacas de esta especie y la calidad de las raíces formadas (Tabla 1). En algunas estacas se observó cierto grado de pudrición de hojas y yemas, aparentemente de origen fisiológico. Al ser una especie que en su entorno natural presenta crecimiento vegetativo y floración durante la época estival, es probable que sea poco tolerante a las condiciones de alta humedad ambiental reinantes en la cama de enraizamiento.

En el segundo ensayo destacó la supervivencia y el enraizamiento de las estacas de Fabiana imbricata tratadas con hormona. Mientras que casi el $80 \%$ de las estacas tratadas sobrevivieron y enraizaron, menos de la mitad de las estacas no tratadas logró enraizar (Tabla 2). Esto indica que $F$. imbricata enraiza fácilmente vía estacas semileñosas tratadas con IBA.

También en el caso de Colliguaja odorifera se observó una mejora significativa en la supervivencia y el enraizamiento de las estacas al tratarlas con hormona enraizante. Sin embargo, se lograron tasas muy bajas de enraizamiento (Tabla 2), no siendo esta una vía recomendable de propagación para la especie.

Por otro lado fue casi nula la respuesta de Baccharis linearis (Tabla 2), incluso en un nuevo intento realizado no se obtuvo enraizamiento de esta especie. Por lo tanto, se concluye que bajo las condiciones ensayadas no es factible propagar Baccharis linearis vía estacas semileñosas. Sin embargo, esta especie se resiembra espontáneamente con mucha facilidad (Riedemann y Aldunate, 2001), siendo probablemente la semilla la forma más factible de propagación.

Los porcentajes de enraizamiento logrados por Escallonia illinita y Fabiana imbricata en solo nueve y doce semanas respectivamente, las perfila como especies capaces de ser instaladas directamente en taludes, vía estacas semileñosas. Villar-Salvador et al. (2011) señalan que es posible utilizar material vegetativo de especies leñosas para la revegetación in situ de taludes, siempre y cuando enraíce fácilmente y la humedad del suelo sea elevada durante un período de tiempo prolongado. Este último requisito se cumpliría durante el período lluvioso en los ambientes mediterráneos. Proustia cuneifolia, que presentó altos porcentajes de supervivencia, posiblemente también sea factible de enraizar directamente en taludes, con la ventaja de ser una especie más tolerante a condiciones de estrés hídrico.

Hartmann et al. (1990) indican que la época óptima para obtención de estacas varía entre especies y está principalmente relacionado con la condición fisiológica del órgano. Baldini (1992) señala que estacas leñosas cosechadas en otoño enraízan mejor que las cosechadas en invierno. En el presente estudio las estacas fueron obtenidas a fines del invierno, por lo que sería recomendable ensayar en la época otoñal las tres especies que mostraron la mayor tasa de supervivencia.

Tabla 2. Efecto de la aplicación de hormona comercial (IBA) sobre el enraizamiento [\%] y la supervivencia [\%] de estacas de Baccharis linearis, Fabiana imbricata y Colliguaja odorifera.

\begin{tabular}{lccc}
\hline \multicolumn{1}{c}{ Especie } & Tratamiento & Enraizamiento & Supervivencia \\
& {$[\%]$} & $2 \pm 2,31$ \\
\hline Baccharis linearis & con hormona & $1 \pm 2,00$ & $1 \pm 2,00$ \\
& sin hormona & $1 \pm 2,00$ & $79 \pm 2,00 \mathrm{a}$ \\
Fabiana imbricata & con hormona & $79 \pm 2,00 \mathrm{a}$ & $48 \pm 10,82 \mathrm{~b}$ \\
& sin hormona & $46 \pm 9,52 \mathrm{~b}$ & $29 \pm 3,83 \mathrm{a}$ \\
& con hormona & $18 \pm 2,31 \mathrm{a}$ & $15 \pm 5,03 \mathrm{~b}$ \\
\hline
\end{tabular}

Los valores seguidos de distintas letras en la columna presentan diferencias estadísticamente significativas, según prueba de Rangos Múltiples de Duncan, con $P \leq 0,05$. 


\section{Conclusiones}

Tanto Escallonia illinita como Fabiana imbricata mostraron ser especies que logran enraizar fácilmente vía estacas semileñosas, y en el caso de la primera sin la necesidad de aplicar hormona de enraizamiento. Estos resultados sugieren que ambas especies podrían ser utilizadas para enraizar directamente en taludes de la zona mediterránea de Chile, instalándolas durante el período de lluvias.

\section{Agradecimientos}

Se agradece la lectura crítica y sugerencias de Flavia Schiappacasse.

\section{Literatura Citada}

Baldini, E.

1992. Arboricultura General. Ed. Mundi-Prensa. España. $380 \mathrm{p}$.

Belmonte, F.; Romero, A.; López, F.; Hernández, E. 1999. Óptimo de cobertura vegetal en relación a las pérdidas de suelo por erosión hídrica y las pérdidas de lluvia por interceptación. Papeles de Geografía, 30: 5-15.

Bochet, E.; García-Palacios, P.; Peco, B.; Toro, J.; García-Fayos, P. 2011. Procesos ecológicos y restauración de la cubierta vegetal. En: F. Valladares, L. Balaguer, I. Mola, A. Escudero, V. Alfaya (Eds.). Restauración ecológica de áreas afectadas por infraestructuras de transporte. Bases científicas para soluciones técnicas. Fundación Biodiversidad, Madrid, España, pp. 101-141.

Brady, N.; Weil, R.

1996. The Nature and Properties of Soils. Prentice-Hall, New Jersey, U.S.A., pp. 563-577.

Etxeberria, M.; Ibáñez, R.

2005. Colonización espontánea de taludes de desmonte sobre margas en Navarra: establecimiento y fuente de diásporas. Publicaciones de Biología, Universidad de Navarra, Serie Botánica, (16): 53-70.

Guevara, R.; Rosales, J.; Sanoja, E.

2005. Vegetación pionera sobre rocas, un potencial biológico para la revegetación de áreas degradadas por la minería de hierro. Interciencia, 30 (10): 644-651.

Gutiérrez, B.

1995. Consideraciones sobre la fisiología y el estado de madurez en el enraizamiento de estacas de especies forestales. Ciencia e Investigación Forestal, 9 (2): 261-277.

Hartmann, H.; Kester, D.; Davies, F. 1990. Plant propagation. Principles and Practices. Fitth Edition. Ed. Prentice-Hall. USA. 697 p.
Honorato, R. 2000. Manual de Edafología. Ed. Universidad Católica de Chile, Santiago, Chile, p. 237-241.

Matesanz, S.; Valladares, F.; Tena, D.; Costa-Tenorio, M.; Bote, D. 2006. Early Dynamics of Plant Communities on Revegetated Motorway Slopes from Southern Spain: Is Hydroseeding Always Needed? Restoration Ecology, 14 (2): 297-307.

Muñoz, M.; Squeo, F.; Leon, M.; Tracol, Y.; Gutiérrez, J. 2008. Hydraulic lift in three shrub species from the Chilean coastal desert. Journal of Arid Environments, 72: 624-632.

Prieto, I.; Padilla, F.; Armas, C.; Pugnaire, F.

2011. The role of hydraulic lift on seedling establishment under a nurse plant species in a semi-arid environment. Perspectives in Plant Ecology, Evolution and Systematics, 13: 181-187.

Riedemann, P.; Aldunate, G.

2001. Flora nativa de valor ornamental, identificación y propagación: Chile Zona Centro. Editorial Andrés Bello, Santiago, Chile, 566 p.

Rondón, J.; Vidal, R.

2005. Establecimiento de la cubierta vegetal en áreas degradadas (principios y métodos). Revista Forestal Latinoamericana, 20 (38): 63-82.

Soto, $\mathrm{M}$.

2003. Catálogo de especies herbáceas y leñosas bajas autóctonas para la revegetacion de zonas degradas en La Rioja. Gobierno de la La Rioja. Consejería de Turismo, Medio Ambiente y Política Territorial. Logroño, España, 61 p.

Villar-Salvador, P.; Soliveres, S.; Quero, J.

2011. Introducción de especies leñosas. En: F. Valladares, L. Balaguer, I. Mola, A. Escudero, V. Alfaya (Eds.). Restauración ecológica de áreas afectadas por infraestructuras de transporte. Bases científicas para soluciones técnicas. Fundación Biodiversidad, Madrid, España, pp. 143-175. 
